\title{
Why Are Males Bad for Females? Models for the Evolution of Damaging Male Mating Behavior
}

\author{
C. M. Lessells ${ }^{*}$
}

Netherlands Institute of Ecology (NIOO-KNAW), P.O. Box 40, 6666 ZG Heteren, The Netherlands

Aвstract: One explanation for the cost to mating for females caused by damaging male mating behavior is that this causes the females to adaptively modify their subsequent life histories in a way that also increases male fitness. This might occur because the reduction in residual reproductive value of the female increases her optimal oviposition rate or because an increase in the current level of damage increases the female's optimal remating interval. In this article, I present models of a stochastic dynamic game in which males choose the level of mating damage that they inflict on females and females choose their oviposition rate and whether to remate. The models show that some level of damage is always an evolutionarily stable strategy and may even provoke females into making terminal reproductive investment (and hence a semelparous life history), that nondamaging populations are always invaded by damaging male mutants, and that damage evolves because of its effect on oviposition rate and despite its effect on remating interval.

Keywords: sexual conflict, cost of mating, manipulation.

Although both males and females gain jointly from reproduction, this process is riddled with sexual conflict (Trivers 1972; Parker 1979; Lessells 1999; Rice 2000; Chapman et al. 2003). This is nowhere more clearly demonstrated than by costs of mating caused by damaging male behavior. Females clearly lose fitness as a result of a cost of mating, so this implies that males must gain either directly or indirectly from such behavior or it would not evolve. Damaging male behavior may include toxins in the seminal fluid (e.g., in Drosophila, Chapman et al. 1995; in the nematode Caenorhabditis elegans, Gems and Riddle 1996) or the imposition of physical damage (e.g., puncture wounds in the female oviduct in Callosobruchus maculatus; Crudgington and Siva-Jothy 2000), but from a theoretical point of view, the evolutionary questions that are raised

\footnotetext{
* E-mail: k.lessells@nioo.knaw.nl.
}

Am. Nat. 2005. Vol. 165, pp. S46-S63. (c) 2005 by The University of Chicago. 0003-0147/2005/1650S5-40815\$15.00. All rights reserved.

are identical. In particular, this behavior is puzzling because in many cases the survival costs to the female that male damage incurs may be paid at least in part while the female is still producing the damaging male's offspring. Any benefit from the damaging behavior must more than compensate for this disadvantage.

Explanations for the damaging male behavior that causes a cost of mating fall into two broad classes. The pleiotropic harm hypothesis (so termed by Morrow et al. 2003) suggests that the cost of mating is not itself selectively advantageous but is the deleterious side effect of some manipulation of female behavior by the male that does increase fitness (Parker 1979). For example, seminal fluid proteins in Drosophila are known to increase oviposition rate, reduce female receptivity, and be involved in removing or destroying the sperm from previous matings (Chen et al. 1988; Harshman and Prout 1994; Price et al. 1999; Chapman et al. 2000; but see Snook and Hosken 2004). These effects clearly increase a mating male's fitness. Perhaps these are the selected benefits of the relevant seminal proteins, and these same proteins also happen to reduce female survival. In contrast, the adaptive harm hypothesis (Morrow et al. 2003) suggests that it is the reduction in survival of the female that the damage causes that is the primary effect and that this invokes consequences that increase male fitness (Constantz 1984; Michiels 1998; Lessells 1999; Johnstone and Keller 2000). Such consequences might come about as a result of phenotypic plasticity in the life history of the female. Damaged females are expected to adaptively modify their life histories to "make the best of a bad job." Nevertheless, the fitness of damaged females will always be lower than that of undamaged females. However, the fitness of the male can be increased, provided that the female modifies her life history in a way that increases the proportion of the damaged female's offspring of which he has paternity. If this increase in paternity more than offsets the decrease in the female's total fitness, damaging male mating behavior will be selected, even if the only direct consequence is to reduce the female's survival. This article explores theoretically whether damaging male mating behavior 
could evolve in the way suggested by the adaptive harm hypothesis.

What sort of modifications of the female's life history might increase a male's fitness? A male's paternity of a female's offspring comes to an end more or less abruptly (depending on the details of sperm competition) when that female remates. A male can therefore gain fitness either if the female increases her reproductive rate in the period when the male has paternity (fig. $1 C$ ) or if she extends the period over which the male has paternity, that is, if she delays remating (fig. 1B). An increase in reproductive rate in response to a decrease in residual reproductive value is a very general prediction of life-history theory (Williams 1966; Charnov and Krebs 1974). It therefore seems plausible to suppose that a male might provoke

(A)

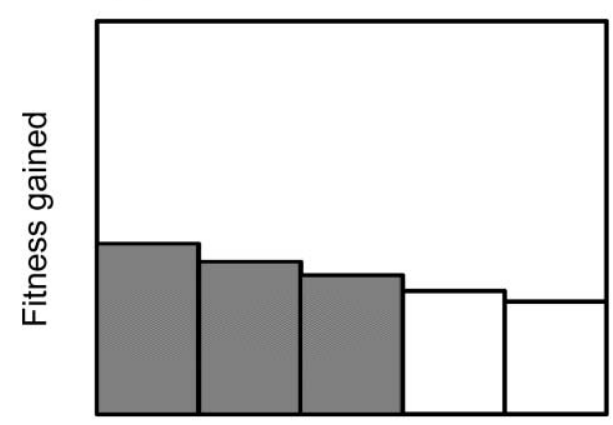

Time

(C)

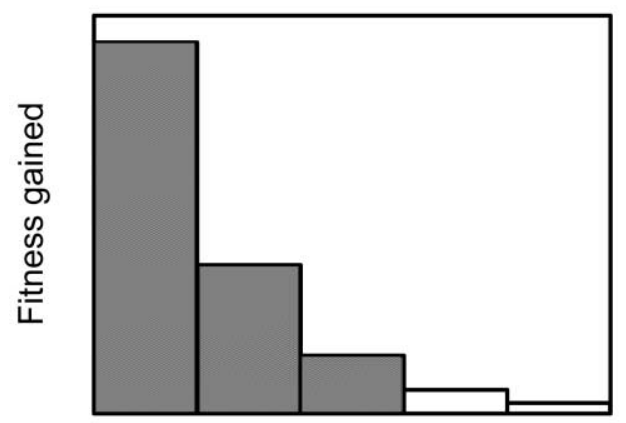

Time a female into increasing her immediate reproduction by reducing her survival prospects (Michiels 1998; Lessells 1999). Similarly, if mating males damage females, alreadydamaged females may delay remating in order to minimize further damage (Constantz 1984; Johnstone and Keller 2000).

The second of these two possibilities-that males gain through damaging mating behavior because females delay remating - has already been considered theoretically by Johnstone and Keller (2000). They focused on male damage through seminal toxins, but, as they point out, their model applies generally to any kind of male mating damage. Their model considers a situation in which females could mate once or twice before any oviposition took place. Females obtained a fecundity benefit by mating a

\section{(B)}

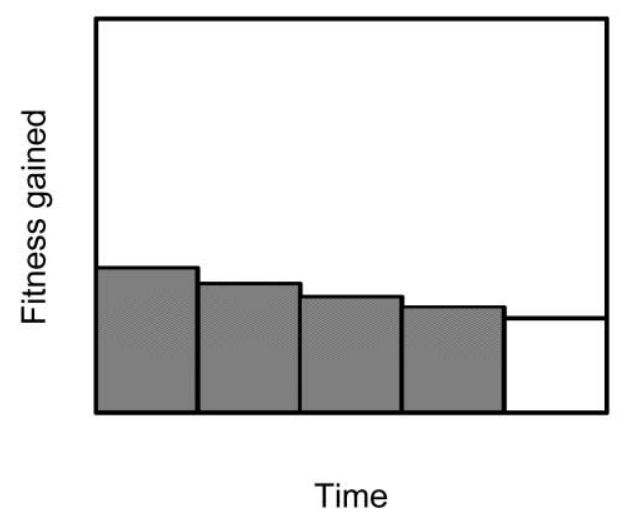

(D)

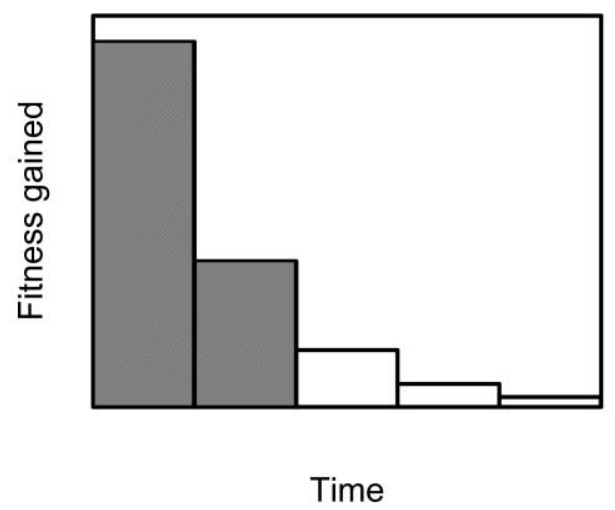

Figure 1: Male damage that is costly to female fitness may be selectively advantageous to the male, provided that it increases that part of the female's fitness of which the male has paternity. Female fitness is the total area of the histogram, but fitness of the first male to mate is the shaded area of the histogram (which indicates the offspring of which he has paternity). Although female fitness is lower in $B$, $C$, and $D$ than in $A$, the reverse is true for male fitness. This can only occur if the male gains paternity for a longer period $(B)$ and/or a higher proportion of the female's reproductive success occurs during the period when he has paternity $(C)$. Male fitness can even increase when one of the two changes is in the adverse direction, provided the other change is strong enough: in $D$, the male has higher fitness than in $A$ because of an increase in the reproductive rate of the female and despite an earlier cessation of paternity. 
second time but also incurred a fecundity cost that depended on the total amount of damage done by the male (or males) with whom they had mated. Under these assumptions, damaging male mating behavior was evolutionarily stable, provided that the cost of damage was an accelerating function of the amount of damage. However, in most cases, nondamaging behavior was an alternative evolutionarily stable strategy (ESS), so that their results implied that an additional explanation for the initial evolution of male damage was needed. Johnstone and Keller's (2000) model serves its purpose in showing that male mating damage can, in principle, be selected in the way that they suggested. However, the life history that the model represents is artificially constrained, with all mating taking place before oviposition and an arbitrary maximum number of matings. Moreover, since there is effectively only one reproductive episode in their model, it considers only adaptive modifications by the female in mating behavior and not in oviposition rate.

In this article, I consider a model of the evolution of male mating damage that does not have these limitations of Johnstone and Keller's (2000) model. First, females can mate repeatedly throughout their reproductive span, so that the maximum number of matings arises as an outcome of the female strategy rather than being a constraint of the model. Second, females make strategic decisions not only about mating but also about their oviposition rate. The main aim of the models was to answer the following questions: If females exhibit adaptive phenotypic plasticity in mating and oviposition behavior in relation to male mating damage, is such damage ever evolutionarily stable? If so, is zero damage also an ESS? In other words, can damaging behavior invade a nondamaging population? And also if so, is the selective advantage of damaging behavior mediated as suggested above by provoking an increase in oviposition rate and remating interval? These questions are answered for a model in which both mating and oviposition rate are female decisions. In addition, the same questions are answered for similar models in which females decide only their oviposition rate or, as in Johnstone and Keller's (2000) model, only whether to remate.

\section{The Model}

In the model, males can inflict damage on the female during mating. The only direct effect of this is to reduce the survival rate of the female. The level of damage inflicted (including whether any damage is inflicted at all) is entirely under the male's control, and inflicting damage incurs no cost to the male. Individual males are assumed to always inflict the same level of damage, as would be expected if they have no cues as to the state of the female, including whether she is a virgin and how much damage she has already suffered. In the initial model, I assume that females have complete control over their oviposition rate and the decision whether to mate. Later in the article, I consider the consequences of females controlling only one of these two variables. Mating is assumed to be costless to females, apart from any damage by the males that they might incur. In other words, there is no cost to either finding a willing male when they decide to mate or repelling males when they decide not to.

The reproductive span of a female is assumed to consist of a number of discrete time steps. In each time step, females decide whether to mate and how many eggs to lay and must then survive to the next time step before repeating these decisions. It is assumed that there is no parental care, so the fitness benefit from eggs once laid is unaffected by how long the female subsequently survives. Initially, I assume that within each time step, mating (if it occurs) takes place before any oviposition. The result of this is that any benefits of mating can accrue to eggs laid in the current time step, whereas the survival costs of mating do not come into play until the end of the current time step. This ordering of mating and oviposition within each time step may have consequences for the evolutionary outcome, and later in the article, I consider the effect of reversing this order.

The model incorporates the following assumptions about the fitness consequences of mating and oviposition: First, damage accumulates each time a female mates and affects survival. The female's level of damage at the end of a time step, $D$, is simply the sum of all damage previously inflicted by males. The survival to the next time step is a function of this level of damage,

$$
s_{D}(D)= \begin{cases}1-\left(D / D_{\max }\right)^{a} & \text { if } D \leq D_{\max } \\ 0 & \text { if } D>D_{\max }\end{cases}
$$

where $D_{\max }$ is the level of damage at which survival to the next time step is 0 , and the constant $a$ determines the way in which survival falls off with increasing levels of damage (see fig. 2A). Because the fitness consequences of damage mean that damage scales to $D_{\max }$, numerical values of inflicted or accumulated damage are given as a proportion of $D_{\max }$ in this article.

Second, there is a cost of reproduction. The model considers oviposition rate as a strategic decision of the female. This will only be the case when there is a cost of reproduction or all females would lay eggs at the same maximum rate determined by physiological constraints. A cost of reproduction is incorporated in the model by assuming that survival to the next time step is a function of the number of eggs laid in the current time step, 
(A)

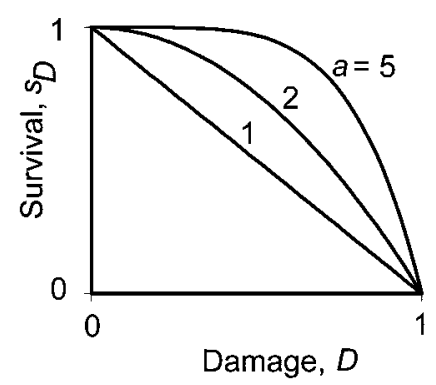

(B)

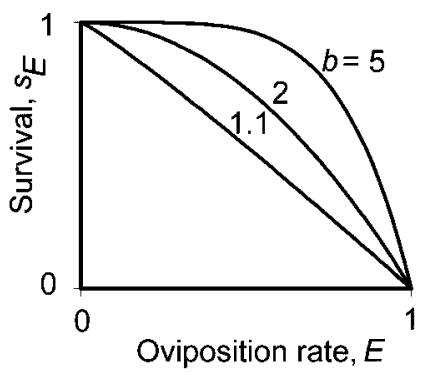

(C)

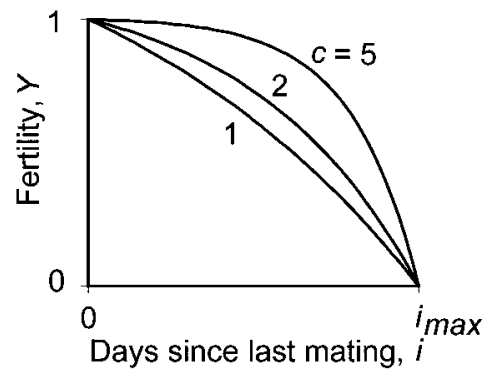

Figure 2: Functions that determine the fitness consequences of mating and oviposition used in the model. $A$, Survival as a function of the accumulated level of damage (eq. [1]). B, Survival as a function of oviposition rate (eq. [2]). C, Fertility of eggs (the proportion fertilized) in relation to the time between last mating and oviposition.

$$
s_{E}(E)= \begin{cases}1-\left(E / E_{\max }\right)^{b} & \text { if } E \leq E_{\max } \\ 0 & \text { if } E>E_{\max }\end{cases}
$$

where $E$ is the number of eggs laid in the time step (the oviposition rate), $E_{\max }$ is the physiologically maximum oviposition rate at which survival to the next time step is zero, and the constant $b$ determines the way in which survival as a function of oviposition rate falls off with oviposition rate (see fig. 2B). Because the fitness consequences of oviposition on survival mean that oviposition rate scales to $E_{\max }$, numerical values of oviposition rate are given as a proportion of $E_{\max }$ in this article.

The overall survival of females from one time step to the next, $s$, is assumed to be the product of the survival in relation to damage and the survival in relation to oviposition rate:

$$
s=s_{D} s_{E}
$$

Third, mating carries benefits as well as costs. The model considers mating as a strategic decision of the female. Since mating carries the cost of damage by males, this will only be the case when there is some benefit to mating, or females would mate only once in their lifetime. In the model, this is incorporated by assuming that the proportion of eggs that are fertilized, $Y$ (fertility), decreases with the number of time units since the female last mated:

$$
Y(i)= \begin{cases}\left\{1-\exp \left[-c\left(i-i_{\max }\right)\right]\right\}\left[1-\exp \left(-c i_{\max }\right)\right] & \text { if } i \leq i_{\max }, \\ 0 & \text { if } i>\mathrm{i}_{\max }\end{cases}
$$

where $i$ is the number of time steps since mating (at the time that the eggs are laid), $i_{\max }$ is the number of time steps at which fertility $Y$ drops to 0 , and $c$ is a constant that determines the rate at which fertility falls off with the number of time steps since mating (see fig. $2 C$ ).

In the model, the fitness of individuals of each sex is taken as the number of fertile eggs of which they are the parent. Females are obviously the parent of all eggs that they lay (and no others). For males, however, paternity depends on the outcome of sperm competition. Here it is assumed that there is complete last male sperm precedence: after mating, the male has paternity of all eggs laid by that female until she mates again. Thereafter he has no paternity via that female, unless he mates again with her.

As a result of the above assumptions, the fitness of males and females depends not only on their own strategy but also on the strategy of individuals of the other sex. The model therefore represents an evolutionary game, and the evolutionary outcome is expected to be a set of ESSs such that neither sex can increase their fitness by changing their strategies. Assessing whether a given level of damage inflicted by males is an ESS can therefore be split into two stages. First, the given damage level is taken as the resident male strategy, and the optimal female mating and oviposition strategies are found. Second, the fitness of male mutants who inflict different levels of damage from the resident males can be assessed in a population of males with the given resident strategy and females with the mating and oviposition strategies that are optimal for that given resident strategy. The resident strategy is an ESS if none of the possible mutants has higher fitness than the resident. Details of how the optimal female strategy and mutant male strategy with the highest fitness were determined are given below. The results of systematic searches considering all possible male strategies as the ESS can be displayed as pairwise invasibility plots (Geritz et al. 1998).

\section{Finding the Optimal Female Strategy for a Given Population Level of Male Damage}

The fitness benefit of a given female strategy depends not only on the population male strategy but also on the state 
of the female in terms of her current level of damage, $A$, and how long, $j$, it has been at the start of the time step since she mated because these influence her survival prospects (and hence residual fitness) and fertility (and hence benefits of current oviposition), respectively. The optimal female strategy is therefore not a single pair of values (mate/do not mate; oviposition rate), but rather a pair of matrices of values for every possible combination of current level of damage and time since the last mating that a female could experience. One matrix, $\mathbf{M}^{*}(A, j)$ contains the optimal mating decision (yes/no), and the other, $\mathbf{E}^{*}(A$, $j$ ), the optimal oviposition rate. Finding the optimal strategy is complicated by the fact that a behavioral decision at one point in time not only affects the current fitness benefit through oviposition and probability of survival but also affects the future state of the female and hence the consequences of future decisions. The solutions to these kinds of problems can be found by stochastic dynamic programming (Mangel and Clark 1988).

The first step in finding the solution is to derive the lifetime fitness function, $F(A, j, t)$, which is the maximum expected fitness that a female can accrue from a given point in time, $t$, during her remaining lifetime, given that her level of damage at the start of the time step is equal to $A$ and the length of time at the start of the time step since she last mated is equal to $j$. This is achieved by assuming that there is some time $T$ beyond which no females survive, solving for $F(A, j, T)$, and then working back through time solving for successively earlier time steps. If all females will die irrespective of their accumulated damage or whether they lay eggs, they should remate (to ensure maximum fertility of the eggs that they lay in this terminal time step) and lay the maximum number of eggs, so that

$$
F(A, j, T)=E_{\max } .
$$

At any earlier time step, $t$, the fitness that a female is expected to accrue during the remainder of her lifetime consists of two terms, the first representing fitness accrued through eggs laid in the current time step and the second fitness accrued through future reproduction. These two terms depend on the female's oviposition rate and whether she remates. Her oviposition rate obviously affects the benefit from current reproduction but also affects her probability of future survival (because of the cost of reproduction). Whether she remates determines both how long it is since she last mated when she lays eggs (and hence her current fertility), her accumulated damage (and hence her future survival), and her future state (and hence her maximum future remaining fitness). If she does not remate, she obtains maximum fitness when she chooses an oviposition rate that maximizes $E Y(j)+s_{E}(E) s_{D}(A) F$
$(A, j+1, t+1)$. If she does remate, she obtains maximum fitness when she chooses an oviposition rate that maximizes $E Y(0)+s_{E}(E) s_{D}(A+d) F(A+d, 1, t+1)$. Whether she should remate depends on which of these is larger; in other words,

$$
\begin{aligned}
F(A, j, t)= & \max \left\{\max _{E_{0}}\left[E_{0} Y(j)+s_{E}\left(E_{0}\right) s_{D}(A) F(A, j+1, t+1)\right] ;\right. \\
& \left.\max _{E_{1}}\left[E_{1} Y(0)+s_{E}\left(E_{1}\right) s_{D}(A+d) F(A+d, 1, t+1)\right]\right\},
\end{aligned}
$$

where $E_{0}$ and $E_{1}$ are the number of eggs laid if the female does not or does remate, respectively. Computationally, the maximum value of each term was calculated by substituting in the optimal clutch size,

$$
E^{*}=\left[Y E_{\max } /\left(b s_{D} V\right)\right]^{1 /(b-1)},
$$

where $V$ is the corresponding value of $F$ at the next time step. (Eq. [7] was found by substituting eq. [2] into the expression $E Y+s_{E}[E] s_{D} V$, taking the partial differential of the resultant expression with respect to $E$ and setting it equal to 0 , and solving for $E=E^{*}$.) After the optimal value of $E$ had been substituted into each of the terms, the maximum value of the two terms was found by calculating both terms and determining which value was numerically larger.

As the dynamic programming equation is solved for successively earlier time steps, the optimal mating and oviposition strategies, and maximum expected fitness, in relation to current damage level and time since last mating converge. This is because the assumptions of the model imply that the fitness consequences of mating and oviposition decisions do not depend on age per se but only on the accumulation of damage that arises from strategic decision making, so that the optimal strategy should, indeed, be independent of age. The optimal mating and oviposition strategies converge on the correct value for a potentially unlimited life span because the values are less and less affected by the assumption that all females die at some arbitrary terminal time step. The mating and oviposition decisions on which the dynamic programming converged were taken as the optimal mating $\left(\mathbf{M}^{*}[A, j]\right)$ and oviposition rate $\left(\mathbf{E}^{*}[A, j]\right)$ strategies. One point that must be emphasized is that they apply uniquely to the population level of damage inflicted by males, $d$, on which they are based.

The stochastic dynamic programming necessitates the choice of the size of increments used in each of the two female state variables (time since mating, $i$, and cumulative damage, $A$ ). The time since mating can only take on values that are an integer multiple of the time step, so the in- 
crement in this state variable must equal the time step chosen. In contrast, the amount of damage inflicted by males, $d$, is a continuous variable. The increment size chosen must be a denominator of $d$. Beyond this, the choice of increment size does not affect the optimal strategy but does affect the precision with which the ESS level of damage can be determined.

\section{Simulating Female Life Histories}

Once the matrices determining optimal oviposition behavior have been determined, they can be used to simulate the life histories of females. Females at the start of their reproductive life span (i.e., at reproductive age $x=1$ ) have never mated, so that the time since last mating at the beginning of the time step, $j_{1}$, is effectively $i_{\max }$ (the time at which fertility becomes 0 ) and the amount of damage that she has accumulated, $A_{1}$, is 0 . At each time step, the mating decision (mate/do not mate) and oviposition rate, $E$, are determined from $\mathbf{M}^{*}(A, j)$ and $\mathbf{E}^{*}(A$, $j)$. After the mating decision has been carried out, the time since last mating and the beginning of egg laying is

$$
i_{x}=\left\{\begin{array}{ll}
j_{x} & \text { if the female has not remated } \\
0 & \text { if she has remated }
\end{array},\right.
$$

and the accumulated damage at the end of the time step is

$$
D_{x}=\left\{\begin{array}{ll}
A_{x} & \text { if she has not remated } \\
A_{x}+d & \text { if she has remated }
\end{array} .\right.
$$

The time since mating at the beginning of the following time step, $j_{x+1}$, equals $i_{x}$, and the amount of accumulated damage at the beginning of the following time step, $A_{x+1}$, equals $D_{x}$. Survival as a function of damage level, $s_{D}$, and survival as a function of oviposition rate, $s_{E}$, can be determined by substituting $D_{x}$ and $E$ into equations (1) and (2), respectively. Survival from time $x$ to $x+1, s_{x}$, is the product of these two separate rates (see eq. [3]). The probability of a female that reaches reproductive age still being alive at age $x$ is

$$
l_{x}=\left\{\begin{array}{ll}
1 & \text { if } x=1 \\
l_{x-1} s_{x-1} & \text { if } x>1
\end{array} .\right.
$$

Fertility, $Y_{x}$, can be determined by substituting $i_{x}$ into equation (4). The number of fertile eggs laid by a female at age $x$ is then $E_{x} Y_{x}$, and the expected output of fertile eggs at age $x$ of a female just reaching reproductive age is $l_{x} E_{x} Y_{x}$. The total expected lifetime output of fertile eggs of a female reaching reproductive age is

$$
\sum_{x=1}^{\infty} l_{x} E_{x} Y_{x}
$$

where $\omega$ is the maximum reproductive span of a female. The probability, $p_{m}$, of a female reaching reproductive age surviving to mate $m$ times is the value of $l$ at the age when females mate for the $m$ th time. If the population of reproductive females has reached a stable age distribution, the probability that a mating female is mating for the $m$ th time is

$$
q_{m}=\frac{p_{m}}{\sum_{m=1}^{z} p_{m}}
$$

where $z$ is the maximum number of times that a female mates.

\section{Finding the Fitness of a Mutant Male Damage Strategy}

The assumptions that males do not pay a fitness cost for the damage that they inflict to females during mating and that the amount of damage inflicted is not conditional on the state of the female imply that a male's expected lifetime fitness is maximized by maximizing the expected fitness from a single mating, $H$. This fitness depends on the male's own damage strategy, $d^{\prime}$, but also, for two reasons, on the resident damage strategy, $d$, of the population males. First, the female mating and oviposition strategies are evolutionarily shaped by the resident damage strategy. Second, the level of damage imposed by the males with the resident strategy interacts on an ecological timescale with the female strategy to generate the female demography derived above. Mutant males are assumed to be sufficiently rare that they do not disturb this average demography. They do, however, influence the subsequent life history of the individual females with whom they have mated. The consequent fitness of mutant males can be obtained by simulations of the female life history like that above. In these new simulations, variables are given two subscripts: the first, $u$, is the time step expressed relative to the time of the mutant mating (when $u=1$ ). The second, $D^{\prime}$, is the accumulated amount of damage after the mating with a mutant male has taken place. Using this convention, $A_{1, D^{\prime}}$ is effectively equal to $D^{\prime}$ and $j_{1, D^{\prime}}$ to 0 . Further simulation of the life history is analogous to that above. Thus, the expected output of fertile eggs in time step $u$ by a female who has damage level $D^{\prime}$ after a mutant mating is $l_{u, D^{\prime}} E_{u, D^{\prime}} Y_{u, D^{\prime}}$. If females lay eggs for $r_{D^{\prime}}$ days before remating and last male sperm precedence is complete, the expected fitness of the male from a mating that results in an accumulated damage level of $D^{\prime}$ is 


$$
\sum_{u=1}^{r_{D^{\prime}}} l_{u, D^{\prime}} E_{u, D^{\prime}} Y_{u, D^{\prime}}
$$

The accumulated level of damage after a mutant mating, $D^{\prime}$, depends on the resident and mutant levels of damage and on how many times the female has already mated. If the mutant mating is the $m$ th mating,

$$
D^{\prime}(m)=(m-1) d+d^{\prime} .
$$

If males encounter females who are willing to mate at random, such females will be mating for the $m$ th time with probability $q_{m}$. Thus, the expected fitness from a single mating of a mutant male with strategy $d^{\prime}$ in a population of males with resident strategy $d$ is

$$
H\left(d^{\prime}, d\right)=\sum_{m=1}^{z} q_{m} \sum_{u=1}^{r_{D^{\prime}(m)}} l_{u, D^{\prime}(m)} E_{u, D^{\prime}(m)} Y_{u, D^{\prime}(m)}
$$

The expected fitness, $H(d, d)$, from a single mating by a resident male can also be obtained from equation (13) by substituting $d^{\prime}=d$.

\section{Results}

\section{Optimal Female Strategies and the Resultant Female Life History}

Stochastic dynamic programming yields optimal female strategies for mating and oviposition rate in relation to their state (time since last mating and cumulative damage) at the start of each time step of the form shown in figure 3. This matrix of optimal female strategies applies uniquely not only to the parameters of the functions that determine the fitness consequences (eqq. [1]-[4]) but also to the level of damage inflicted by the population males, $d$ (in the case shown in fig. $3, d=0.1$ ). Each square of the matrix corresponds to a given female state and indicates whether the female should mate (indicated by bold entries in the shaded area of the matrix) and how many eggs to lay in the time step (given as a proportion of the physiologically maximum oviposition rate, $E_{\max }$ ). One general feature of the optimal oviposition rates is that for a given time since last mating, oviposition rate increases with accumulated damage (i.e., the values increase down each of the columns in the oviposition strategy matrix). This follows from equation (7) because both $s_{D}$ and the remaining fitness at the next time step decrease with the amount of accumulated damage, while fertility, $Y$, is constant for a given number of days since mating.

The female life history generated by a particular strategy

\begin{tabular}{|c|c|c|c|c|c|c|}
\hline \multirow[b]{2}{*}{0} & 0 & \multicolumn{4}{|c|}{ Time since mating } & \multirow{2}{*}{0.160} \\
\hline & 0.117 & 0.113 & 0.088 & 0.035 & 0.002 & \\
\hline & 0.160 & . & . & $0.051)$ & 0.251 & 0.251 \\
\hline & 0.251 & 0.258 & 204 & 0.433 & 0.433 & 0.433 \\
\hline & 0.433 & 0.433 & 313 & 0.747 & 0.747 & 0.747 \\
\hline & 0.747 & 1.000 & 1.000 & 1.000 & 1.000 & 1.000 \\
\hline & 1.000 & 1.000 & 1.000 & 1.000 & 1.000 & 1.000 \\
\hline & 1.000 & 1.000 & 1.000 & 1.000 & 1.000 & 1.000 \\
\hline & 1.000 & 1.000 & 1.000 & 1.000 & 1.000 & 1.000 \\
\hline & 1.000 & 1.000 & 1.000 & 1.000 & 1.000 & 1.000 \\
\hline & 1.000 & 1.000 & 1.000 & 1.000 & 1.000 & 1.000 \\
\hline & 1.000 & 1.000 & 1.000 & 1.000 & 1.000 & 1.000 \\
\hline
\end{tabular}
matrix can be found by simulating the life history. At the beginning of her reproductive span, the female has no
Figure 3: Example of the optimal female strategy generated by the model using stochastic dynamic programming. Numbers in the table are the optimal oviposition rate for a female in relation to her state (accumulated level of damage and time since last mating). For females in the shaded area with oviposition rates in bold, the optimal strategy is to mate before laying. Increments in time since remating are single time steps, and those in damage are 0.1 (of the maximum level of damage) — the damage level of population males for which this matrix was calculated. Females entering their reproductive span have zero damage and are the equivalent of $i_{\max }$ time steps after mating (because they are infertile). Their optimal strategy is therefore that circled at top right. One time step later they are therefore in the left-hand state circled in the next row. In subsequent time steps they move along each row until they remate, when they move down to the next row. This matrix was calculated assuming $d=0.1$, $i_{\max }=5, a=3, b=1.1$, and $c=5$. (Note that, although the female strategy is optimal for this level of male population damage, the pair of male and female strategies is not an evolutionarily stable strategy because mutant males inflicting 0.45 damage have the highest fitness.)

accumulated damage $(A=0)$, and because she has not mated, she has zero fertility, which is equivalent to a time since last mating of 5 ( $i$ effectively equals 5 ). Her state therefore corresponds to the top right cell that is circled in figure 3. She therefore remates and lays eggs at a rate of 0.160 (these rates are expressed as a proportion of the physiologically maximum oviposition rate). Her fertility and output of fertilized eggs in the current time step and survival to the next are determined by the mating and oviposition decisions. These decisions also affect her state in the next time step. Because she has mated, the time since last mating at the beginning of the next time step is 1 , and the amount of damage she has accumulated is incremented by the amount of damage done by population males, $d$, which is 0.1 in this case. Her state at the next time step therefore corresponds to the next circled cell in 
figure 3. Thus, she should lay eggs at a rate of 0.161 without remating first. Output of fertilized eggs and survival and state in the following time step can be calculated as before. In this way the whole of the life history can be simulated. Because the only stochasticity in the model is in survival, all surviving females follow the same life-history trajectory.

The life history simulated from the strategy matrix in figure 3 is shown in figure 4. In this particular example, the parameters of the functions determining fitness consequences (eqq. [1]-[3]) were such that survival and fertility remain rather constant and then drop off suddenly with increasing accumulated damage, $D$, and days since mating, $i$, respectively $(b=3, c=5)$, whereas survival drops off rather gradually with oviposition rate $(a=1.1)$. This accounts for the relative magnitude of the effects of these different kinds of fitness consequences, which might be reversed with other parameter values. Figure $4 A$ shows the mating and oviposition decisions and also the consequent damage accumulated by the female. Females remate at decreasing intervals throughout their reproductive span. In this example, females remate at least one time step before they would become totally infertile, emphasizing that remating is a strategic decision balancing the costs and benefits of mating. Within each mating interval, oviposition rate tends to rise slightly and then fall. This is the outcome of the value of current oviposition and residual fitness both falling, but at different rates. The value of current reproduction falls because of decreasing fertility. Residual fitness falls because of the reduction in fertility of eggs laid in the remaining time steps before remating
(A)

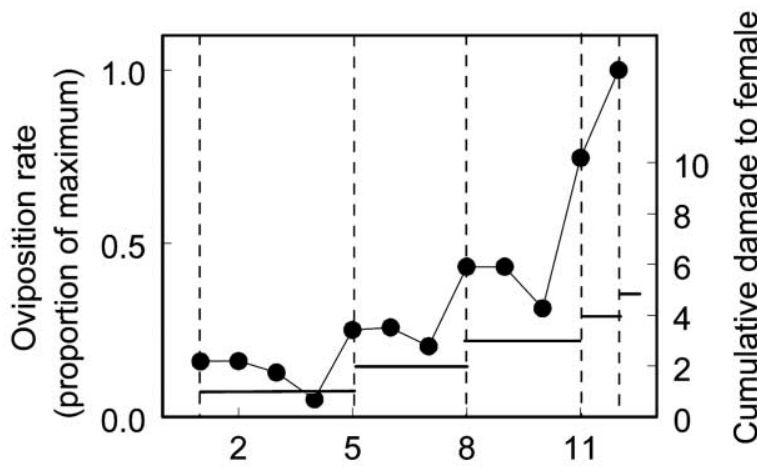

Female reproductive age (time steps)

(C)

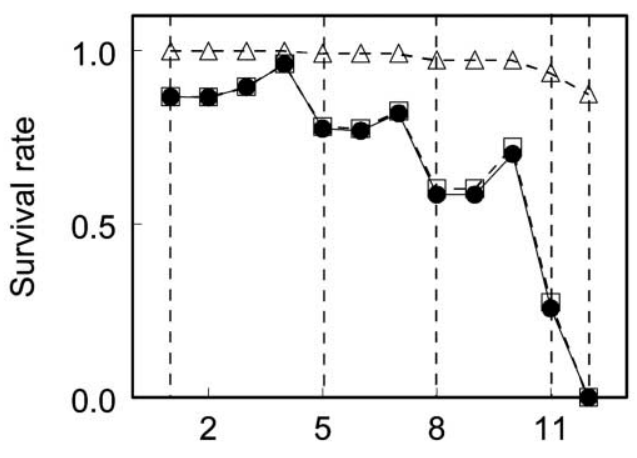

Female reproductive age (time steps)
(B)

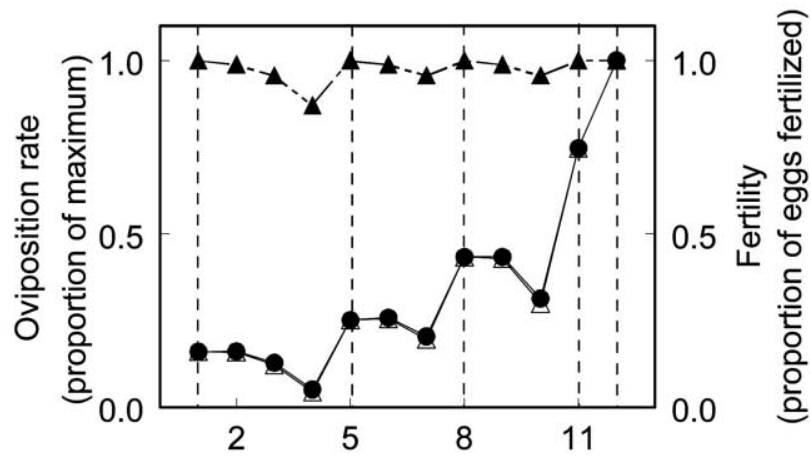

Female reproductive age (time steps)

(D)

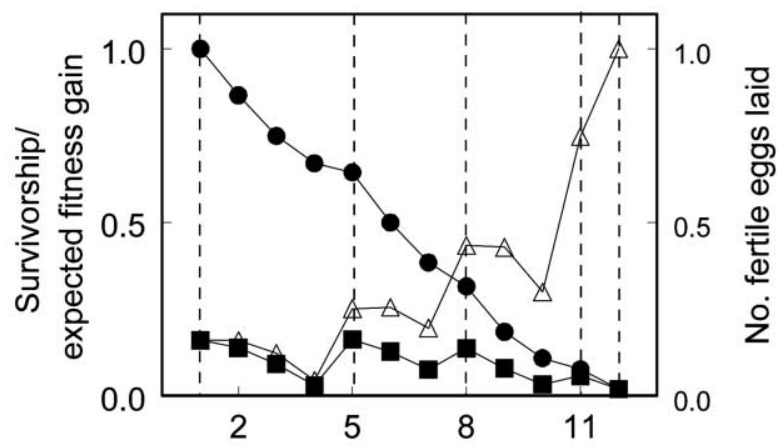

Female reproductive age (time steps)

Figure 4: Example of the female life history produced by simulation from the optimal mating and oviposition strategy. The vertical dashed lines indicate when remating occurs. A, Oviposition rate (filled circles) and cumulative damage (horizontal lines). B, Oviposition rate of all eggs (filled circles) and of fertile eggs (open triangles) and fertility (filled triangles). $C$, Survival as a function of damage, $s_{D}$ (open triangles), survival as a function of oviposition rate, $s_{E}$ (open squares), and overall survival rate, $s$ (filled circles). D, Survivorship (filled circles), the number of fertile eggs laid (open triangles), and the expected fitness gain (filled squares). The life history shown here is generated from the strategy matrix given in figure 3 , which was calculated assuming $d=0.1, i_{\max }=5, a=3, b=1.1$, and $c=5$. 
and an increasingly imminent increase in damage as a result of remating. The oviposition rate in the time step in which a female mates becomes higher with successive matings. This happens because the female's residual fitness is lower because of the accumulated damage. Eventually, females make a terminal reproductive investment by laying the physiologically maximum number of eggs leading to certain death before the next time step. (This terminal investment is a general feature of all optimal female life histories except when the population males are nondamaging and females are, as a result, potentially immortal. However, the proportion of females that reach the age at which this terminal investment occurs is usually small.) In this particular example, females make this terminal investment long before the level of accumulated damage approaches that leading to certain death. Fertility drops, of course, within each remating interval. In this particular example, females remate before fertility has dropped much, so that the output of fertilized eggs is scarcely lower than the oviposition rate (fig. $4 B$ ). The two components of survival change in different ways during the females' reproductive span (fig. $4 C$ ). Survival as a function of damage $\left(s_{D}\right)$ must decrease monotonically, but the survival as a function of oviposition rate $\left(s_{E}\right)$ mirrors the changes in oviposition rate and thus shows some short-term increases over successive time steps superimposed on a falling trend. In this particular example, survival as a function of oviposition rate is the major determinant of overall survival between time steps (but again this might not be the case with other parameter values). Finally, figure $4 D$ shows the fitness gained by females in each time step. Fitness is measured in terms of fertile eggs laid $(E Y)$, which tends to increase through the female's reproductive span. However, females only gain this fitness if they survive until that time. For a female just beginning reproduction, her expected fitness gain in each time step must take into account the probability that she is still alive (survivorship, $l$ ). Her expected fitness is the product of these two components $(E Y l)$ and is much more constant over the reproductive span (fig. $4 D$ ). With the life history shown in figure $4 D$, the expected number of times that a female mates is 2.054 .

\section{Mutant Male Strategies with the Highest Fitness}

Once the life history of females has been derived, this information can be used to derive the fitness that a male can expect from mating with a female. This fitness depends only on the level of accumulated damage after mating has taken place. Figure 5 shows these expected fitness gains to a male for individual time steps after mating takes place for five different levels of accumulated damage. The fitness gained at each time step depends on the female's choice of oviposition rate and the number of time steps for which

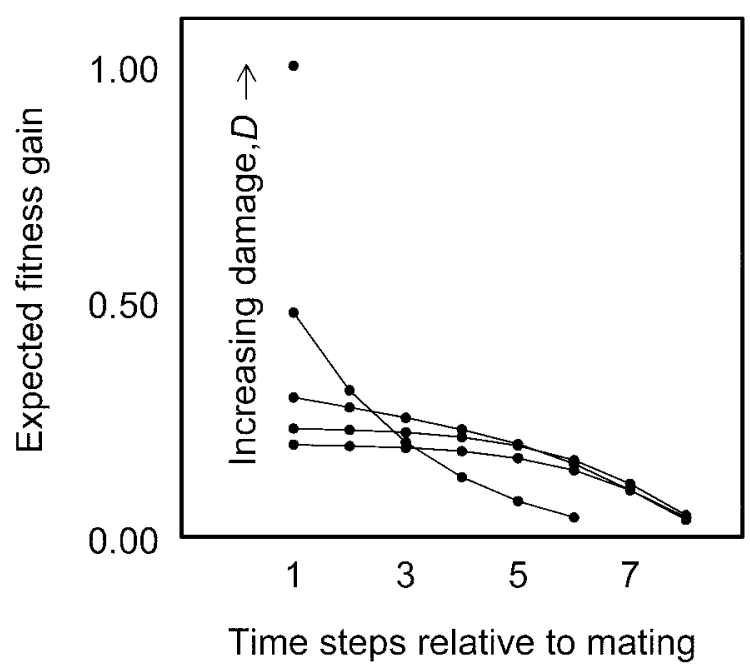

Figure 5: Example of the expected fitness gains for a male from mating with a female in relation to the accumulated damage of the female after mating. From bottom to top, the left-hand ends of the lines and the point refer to accumulated damage levels of $0.1,0.3,0.5,0.7$, and 0.9 . The figure shows how the expected fitness gain are distributed across time after laying. The respective total expected fitness gains are 1.160, $1.358,1.522,1.232$, and 1.000 . Values shown are generated from a model assuming $d=0.3, i_{\max }=8, a=5, b=2$, and $c=5$.

the male gains fitness (i.e., the length of the curves in fig. $5)$ on the female's decision when to remate. The total expected fitness of the male is the sum of all the points on the relevant line. The figure shows that, while the oviposition rate in the time step in which mating takes place always increases with the accumulated level of damage after mating, total male fitness also depends on how fast fitness gains fall off with days after mating (because of female mortality and decreasing fertility) and how long the female waits before remating. In figure 5, the total expected fitness of males increases and then decreases again with increasing accumulated damage after mating (see the legend to fig. 5). Although each male is assumed to inflict the same damage each time he mates, this does not result in all females having the same level of accumulated damage after mating because females will have sustained varying levels of damage before mating, depending on the damage inflicted by population males and the number of times they have already mated. The expected fitness of a male from a mating therefore depends on this distribution of damage that females have already sustained, as well as the male's own level of damage (see eq. [13]). Figure 6 shows the expected fitness of a mating in relation to the male's own level of damage $\left(d^{\prime}\right)$ for four different resident population levels of damage (parameter values are the same in figs. 5, 6). All four of these rela- 
(A)

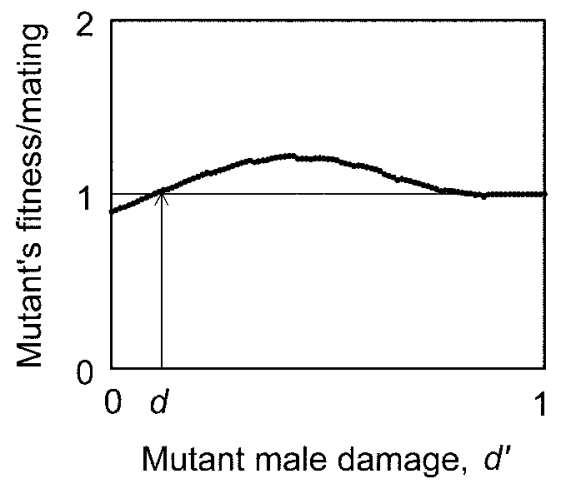

(C)

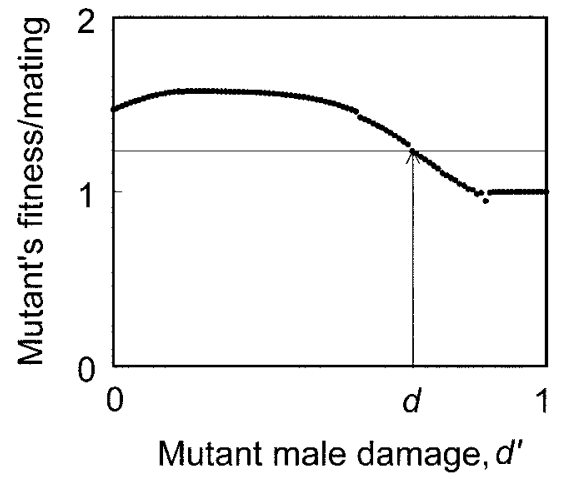

(B)

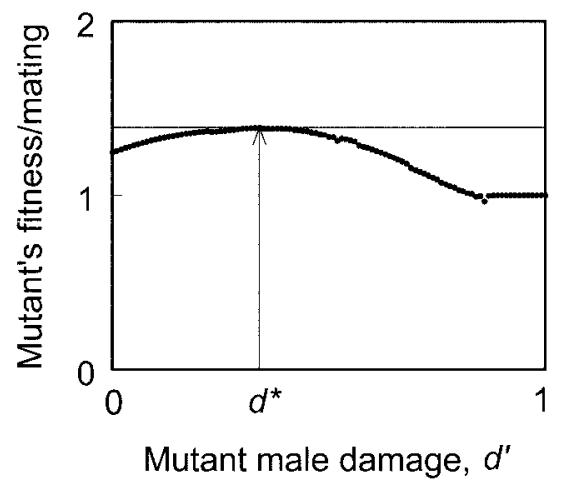

(D)

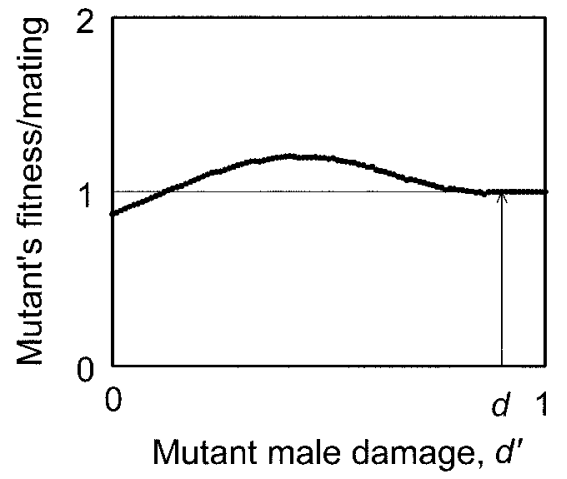

Figure 6: Finding the evolutionarily stable strategy (ESS) male damage strategy: mutant fitness in relation to mutant damage level. Any mutants with fitness higher than that of the resident male damage strategy, $d$ (indicated by a horizontal line), will invade. Only when the resident male strategy has the highest fitness $(B)$ is that strategy an ESS. Parameter values are the same as for figures 5 and $7 A$ : $i_{\max }=8, a=5, b=2$, and $c=5$. Values of $d$ are as follows: for $A, 0.10$; for $B, 0.34$ (the ESS); for $C, 0.70$; for $D, 0.90$.

tionships show a peak, but that is not always the case for different population parameters.

\section{Evolutionarily Stable Male Damage and Female Oviposition and Mating Strategies}

Relationships between mutant fitness and mutant level of damage like those shown in figure 6 reveal whether the resident damage level to which they refer is evolutionarily stable. Each of the figures indicates the fitness of the resident males with a horizontal line. Any mutants that have a fitness higher than this will invade the resident population, so the resident strategy is only the ESS when the mutant strategy identical to the resident strategy has the highest fitness (fig. $6 \mathrm{~B}$ ). More general stability properties can be investigated by considering resident strategies other than the ESS. For the example shown in figure 6, if the resident strategy is below the ESS (e.g., fig. 6A), mutants that inflict more damage will invade, while the reverse is true above the ESS (e.g., fig. $6 C, 6 D$ ). The fitness of all mutants can be shown for a comprehensive range of resident strategies in a pairwise invasibility plot (e.g., fig. $7 \mathrm{~A}$, which corresponds to figs. 5, 6). Since all that determines whether a mutant strategy will invade is its fitness relative to the resident males, this is all the plots need to show. This is done on the plots by shading black areas where the mutant has higher fitness than the residents and leaving unshaded the areas where the residents have higher fitness. In the plots shown in this article, areas where the mutant's and resident's fitnesses are equal are shaded gray. Because the mutant's strategy is plotted on the $Y$-axis and the resident's strategy on the $X$-axis, vertical strips in the pairwise invasibility plots correspond to the $X$-axis in plots like those in figure 6 , and the mutants necessarily have the same fitness as the residents along the major diagonal (because their strategies are identical). The ESSs are represented by vertical strips in which only the diagonal cell is shaded. The population will converge on the ESS if the 
(A)

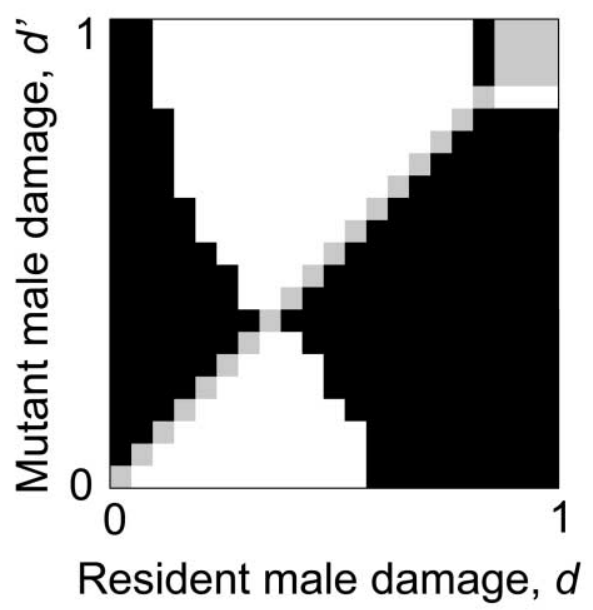

(B)

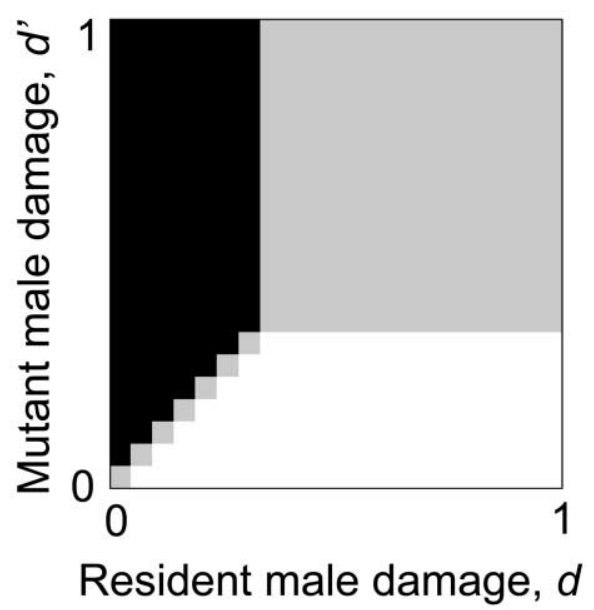

Figure 7: Finding the evolutionarily stable strategy (ESS) male damage strategy: pairwise invasibility plots. Vertical strips in these figures correspond to the $X$-axis in plots like those in figure 6. ( $A$ is for the same parameter values as in fig. 6.) Black shading indicates areas where the mutant has higher fitness (points above the horizontal line indicate the fitness of residents in fig. 6), white shading indicates areas where the resident has higher fitness (points below the horizontal line in fig. 6), and gray shading indicates where the mutant and residents have equal fitness (points on the line in fig. 6). A resident male strategy is an ESS when the corresponding vertical strip contains no black shading (all mutant strategies have equal or lower fitness). A, An ESS with an intermediate level of male damage. Parameter values are the same as for figures 5 and $6: i_{\max }=8, a=5, b=$ 2, and $c=5$. B, An ESS with terminal damage. Below a certain level of resident male damage, all points above the diagonal are black, and all below are white, indicating that any mutants inflicting higher damage than the residents will invade. Above this resident damage level, all mutants inflicting higher damage are equally fit, and those inflicting less damage have lower fitness. The ESS lies in this upper range of damage levels. (All of these damage levels are equivalent because all provoke females to make terminal investment.) Parameter values for $B$ are: $i_{\text {max }}=8, a=2, b=1.1$, and $c=1$. In $A$ and $B$, the left-hand column (for resident male damage $d=0$ ) is black apart from the diagonal square, indicating that any damaging mutant will invade a nondamaging population.

plot is black above and white below the diagonal to the left of the ESS and the reverse to the right. Other stability properties that can be diagnosed from pairwise invasibility plots are explained by Geritz et al. (1998). In the plots for the model presented in this article, there is a square area in the top right-hand corner where the mutants and resident have the same fitness. This occurs because both mutants and residents in this area inflict a level of damage that causes females to make terminal reproductive investment. Another feature of the plots for the model presented here is that they show irregularities (e.g., the white bar in the top right of fig. 7A). These are due to the discrete time steps and are generally small (e.g., the white bar in fig. $7 \mathrm{~A}$ can be seen as a tiny drop below the fitness of the resident males just to the left of the arrow indicating the resident male fitness in fig. $6 D$ ), although they can be larger. These irregularities would be smoothed out by stochastic variation in, for example, the damage inflicted by males and have been ignored in the results presented below.

In the models, some level of damage greater than 0 is always the ESS. In some cases, this level of damage results in the female life history being iteroparous. For example, under the ESS shown in figure $6 \mathrm{~B}$, females reproduce for up to 16 time steps and mate up to three times. However, not all parameter values give ESSs with such intermediate levels of damage. In some cases, the evolutionarily stable level of damage is such that the females make a terminal reproductive investment. As a result, females are semelparous, mating only once and ovipositing and then dying before the next time step. Figure $7 B$ shows a pairwise invasibility plot when the ESS represents such "terminal damage."

Is zero damage ever an ESS in addition to the ESS with intermediate or terminal damage? Or, put another way, are damaging mutants able to invade a nondamaging male population? In such a benign population, there is no cost to females of mating, so because there is a fertility benefit to mating, females should mate at every time step. With last male sperm precedence, males then only gain paternity in the time step in which they mated with the female (unlike in fig. 5, where females do not mate in every time step). Since female oviposition rate always increases with her level of accumulated damage for any given time since last mating, the male will maximize his fitness if he max- 
imizes the female's damage. In other words, damaging mutants will always invade a nondamaging population.

Table 1 shows how the parameters $a, b$, and $c$ determining the fitness consequences affect the value of the ESS. The parameter $a$ determines the curvature of the function relating survival as a function of damage to the accumulated level of damage (fig. $2 A$ ). Evolutionarily stable levels of damage tend to increase as $a$ increases; that is, the effect of damage on survival becomes more strongly accelerating. A similar phenomenon occurred in Johnstone and Keller's (2000) model: toxicity was only an ESS when it had an accelerating effect on fitness. They attributed this to females being less willing to remate and risk further damage when this would have a disproportionate effect on their fitness. This explanation implies that damage produces its effect by delaying remating, but the reverse occurs in the model presented here. A second difference from Johnstone and Keller is that zero damage is never an ESS because damaging males will always invade a nondamaging population. Thus, damage is an ESS even if damage has a decelerating effect on fitness (i.e., $a<1$ ).

The parameter $b$ determines the curvature of the function defining the cost of reproduction (fig. $2 B$ ). The lowest evolutionarily stable values of damage occur with the highest values of $b$, and terminal damage is the ESS when $b$ is reduced to close to 1 . This is because the fitness differentials between different oviposition rates increase with the degree of curvature, so when the curvature is slight, the detrimental effect of damage on future reproduction can easily tip the balance in favor of current reproduction.

The parameter $c$ affects how quickly fertility falls off with increasing time since mating (fig. 2C). For the parameter combinations explored in table 1, this appears not to have a strong effect on the evolutionary outcome.

Does damage increase male fitness in the way suggested in the introduction, that is, by provoking females into increasing their oviposition rates and extending the interval before remating? As noted above, oviposition rates do increase with increasing levels of damage. However, in the model presented here, remating intervals (the number of entries in a row in the female strategy matrix before remating) always remain constant or decrease with increasing damage (see, e.g., fig. 3). This occurs because increasing damage lowers residual fitness, and ensuring the fertility of current eggs by remating becomes relatively important compared with the detrimental effects of further damage. Thus, damage by males evolves because of its effect on female oviposition rates and despite its effect on the remating interval.

\section{Variations on the Initial Model}

\section{Females Control Oviposition Rate but Not Mating}

In the above model, females make strategic decisions as to whether to mate and how many eggs to lay. In this section, I consider the evolutionary outcome when females retain control over their oviposition rate but mating is no longer under their control and occurs with a probability $k$ at the beginning of each time step. Under these assumptions, the terminal fitness equation is

$$
F(A, j, T)=(1-k) E_{\max } Y(j)+k E_{\max } Y(0),
$$

Table 1: Evolutionarily stable values of male damage in relation to parameter values affecting the fitness consequences of mating and oviposition

\begin{tabular}{|c|c|c|c|c|c|c|c|c|c|c|}
\hline \multirow{2}{*}{$\begin{array}{l}\text { Mating before/after } \\
\text { oviposition }\end{array}$} & \multirow[b]{2}{*}{$b$} & \multicolumn{3}{|c|}{$a=1$} & \multicolumn{3}{|c|}{$a=2$} & \multicolumn{3}{|c|}{$a=5$} \\
\hline & & $c=1$ & $c=2$ & $c=5$ & $c=1$ & $c=2$ & $c=5$ & $c=1$ & $c=2$ & $c=5$ \\
\hline Before & 1.1 & $.10^{\mathrm{a}}$ & $.10^{\mathrm{a}}$ & $.10^{\mathrm{a}}$ & $.35^{\mathrm{a}}$ & $.35^{\mathrm{a}}$ & $.35^{\mathrm{a}}$ & $.65^{\mathrm{a}}$ & $.65^{\mathrm{a}}$ & $.65^{\mathrm{a}}$ \\
\hline Before & 2 & $?$ & $<.05$ & .05 & $(.15)$ & .15 & .15 & .45 & .40 & .35 \\
\hline Before & 5 & $<.05$ & $<.05$ & $<.05$ & $<.05$ & $<.05$ & $<.05$ & .10 & .10 & .10 \\
\hline After & 1.1 & .05 & .05 & .05 & .15 & .15 & .15 & .45 & .45 & .40 \\
\hline After & 2 & $<.05$ & $<.05$ & $<.05$ & .15 & .15 & .15 & .35 & .35 & .35 \\
\hline After & 5 & $<.05$ & $<.05$ & $<.05$ & $<.05$ & $<.05$ & $<.05$ & .10 & .10 & .10 \\
\hline
\end{tabular}

Note: The variable $a$ determines the rate at which survival declines with increasing accumulated damage to females (see eq. [1]; fig. $2 A$ ); $b$ determines the rate at which survival declines with increasing current oviposition (see eq. [2]; fig. 2B); and $c$ determines the rate at which fertility declines with increasing time since mating (see eq. [4]; fig. 2C). Evolutionarily stable values were determined from pairwise invasibility plots with damage strategies of mutants considered at intervals of 0.05 (as in the figures). Parentheses indicate that the stability properties were unclear, and a question mark indicates that the evolutionarily stable strategy (ESS) could not be determined from the pairwise invasibility plots.

a The ESS was for terminal damage. In these cases, all higher values of damage are also ESSs. 


$$
\begin{aligned}
F(A, j, t)= & \max _{E_{0}}\left[(1-k)\left(E_{0} Y(j)+s_{E}\left(E_{0}\right) s_{D}(A) F(A, j+1, t+1)\right]\right. \\
& +\max _{E_{1}}\left[k\left(E_{1} Y(0)+s_{E}\left(E_{1}\right) s_{D}(A+d) F(A+d, 1, t+1)\right] .\right.
\end{aligned}
$$

There is also now only one matrix specifying the female strategy, which contains the optimal oviposition rate in relation to the damage level and time since mating (after any mating has taken place), $\mathbf{E}^{*}(D, i)$. Under this new model, females no longer all mate at the same times during their reproductive span. As a result, individual females may follow different life-history trajectories while implementing the same optimal strategy. This means that when the life history is simulated, the simulation must keep track of the proportion of the females in each possible state and not simply the state of all females. In other respects, the calculations are the same as for the first model.

The results are broadly similar to those of the first model: intermediate levels of damage (fig. 8) or terminal damage may be the ESS, with terminal damage being more likely the more linear the function for the cost of reproduction (the lower the value of $b$ ); damaging males always invade a nondamaging population; and optimal oviposition rates for a given time since last mating (measured after any mating may have taken place within the time step) always increase with damage level (after any mating within the time step). The major difference is that the fitness differentials between males inflicting different levels of damage are much smaller (see fig. $8 B$ ). This probably results from stochastic variation in the remating intervals of females generated by females having a constant prob- ability of mating in each time interval, irrespective of their damage level or time since previous mating.

\section{Females Control Mating but Not Oviposition Rate}

The previous section considers a model in which females control oviposition rate but not mating. This section considers the inverse scenario: females control mating but not oviposition rate. Instead, females all lay eggs at the same constant rate $E$. The terminal fitness equation is then

$$
F(A, j, T)=E,
$$

and the dynamic programming equation is

$$
\begin{aligned}
F(A, j, t)= & \max \left\{\left[E Y(j)+s_{E}(E) s_{D}(A) F(A, j+1, t+1)\right] ;\right. \\
& {\left.\left[E Y(0)+s_{\mathrm{E}}(E) s_{D}(A+d) F(A+d, 1, t+1)\right]\right\} . }
\end{aligned}
$$

Under the optimal remating strategy, females all follow the same life-history trajectory, so simulating the life history is done in the same way as for the first model.

Under the assumptions of the new model, intermediate damage is always an ESS. Terminal damage is never an ESS because female oviposition rate is fixed, so there is no possibility for the males to provoke the females into terminal reproductive investment. Also, damaging mutants no longer invade a nondamaging population. Instead, they are selectively neutral (see left-hand column in fig. $9 A$ ). The reason for this is that, in a nondamaging population, females always mate at every time step because
(A)

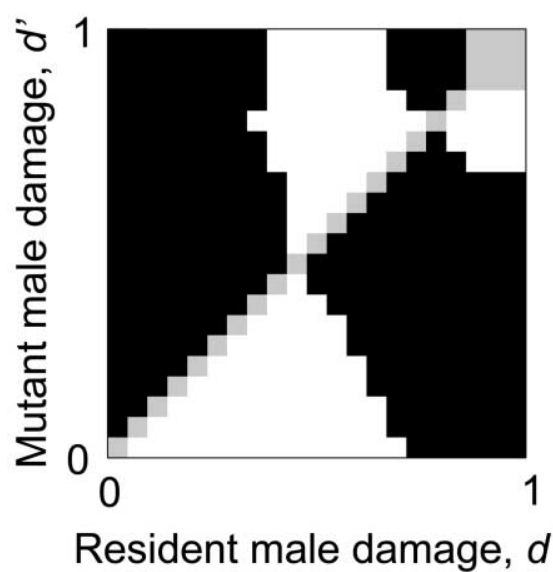

(B)

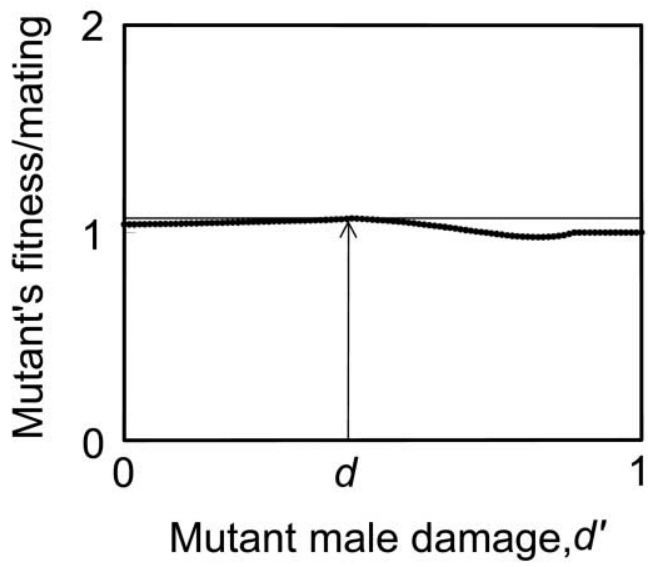

Figure 8: Evolutionarily stable strategy male damage in a model in which females decide oviposition rate but mate with a fixed probability each time step. Parameter values are $k$ the probability of mating per time step $=0.2, i_{\max }=8, a=5, b=2$, and $c=5$. 
(A)

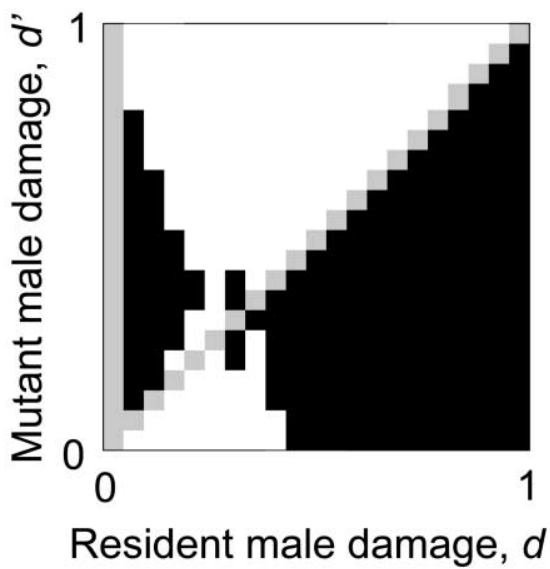

(B)

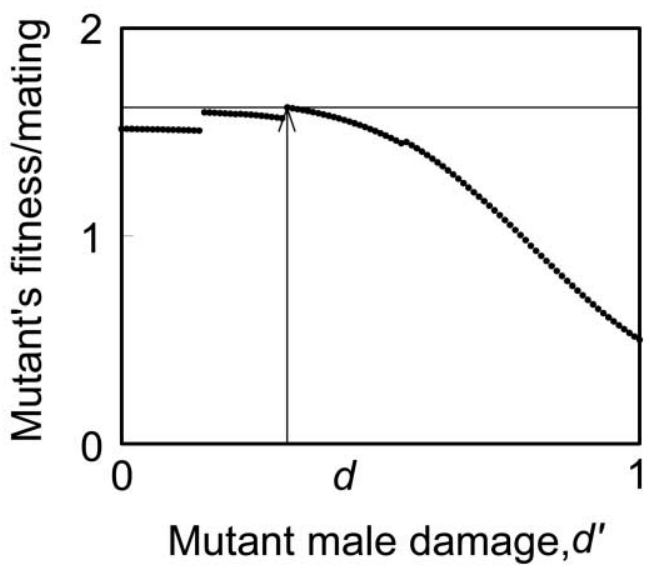

Figure 9: Evolutionarily stable strategy male damage in a model in which females decide whether to mate but oviposit at a fixed rate. Parameter values are $E$ the oviposition rate $=0.5, i_{\max }=8, a=5, b=2$, and $c=5$.

there is no cost to mating. With complete last male sperm precedence, this implies that a male only gains fitness from the eggs that the female lays on the day on which he has mated with her. Thus, a female from a nondamaging population who is mated by a damaging mutant male lays the same number of eggs, and that damaging mutant has the same fitness as would a nondamaging male. However, damaging mutants would be subject to drift, and as soon as the population males inflict some small amount of damage, damaging mutants can become selected (see fig. 9A). Another major difference from the outcome of the model in which females also decide their oviposition rate is in the remating interval (fig. 10). In the model in which both mating and oviposition are female decisions, the optimal female strategy always results in remating interval decreasing with increasing levels of accumulated damage (whether the resident male damage level is the ESS). In contrast, in the model in which females decide only whether to mate, the evolutionarily stable female strategy always results in remating intervals first increasing and then decreasing with increasing levels of accumulated damage. Indeed, it has to be true that remating intervals increase over at least part of the range or damage could never be an ESS because, given that female oviposition rates are fixed in this model, increasing remating intervals is the only way in which males can gain by damaging females.

\section{Within Each Time Step, Oviposition Occurs before Mating}

In all of the models above, mating is assumed to occur before oviposition within each time step. The result is that the fertility benefits of remating are experienced for one time step before any survival cost of the damage thus incurred are paid. Here I consider the consequences of oviposition occurring before mating, so that the reverse is true. Under these new assumptions, the terminal fitness function is

$$
F(A, j, T)=E_{\max } Y(j)
$$

and the dynamic programming equation is

$$
\begin{aligned}
F(A, j, t)= & \max _{E_{0}}\left\{\max _{0}\left[E_{0} Y(j)+s_{E}(E) s_{D}(A) F(A, j+1, t+1)\right] ;\right. \\
& \left.\max _{E_{1}}\left[E_{1} Y(j)+s_{\mathrm{E}}\left(E_{1}\right) s_{D}(A+d) F(A+d, 1, t+1)\right]\right\} .
\end{aligned}
$$

One consequence of the reversal of the behavior sequence within a step is that the minimum interval between mating and oviposition is 1 , not 0 . In order that the benefits of mating for fertility be numerically comparable between this and the earlier model, the value of $i_{\max }$ was set one higher than in runs of the earlier model with otherwise identical parameters, and fertility was calculated as

$$
Y(i)= \begin{cases}\left\{1-\exp \left[-c\left(i-i_{\max }\right)\right]\right\}\left\{\left\{1-\exp \left[-c\left(i_{\max }-1\right)\right]\right\}\right. & \text { if } i \leq i_{\max } \\ 0 & \text { if } i>i_{\max }\end{cases}
$$

The result of these modifications is that the form of the decrease in fertility with time since last mating is identical but occurs one time step later.

This model, like the first model with the reverse ordering 


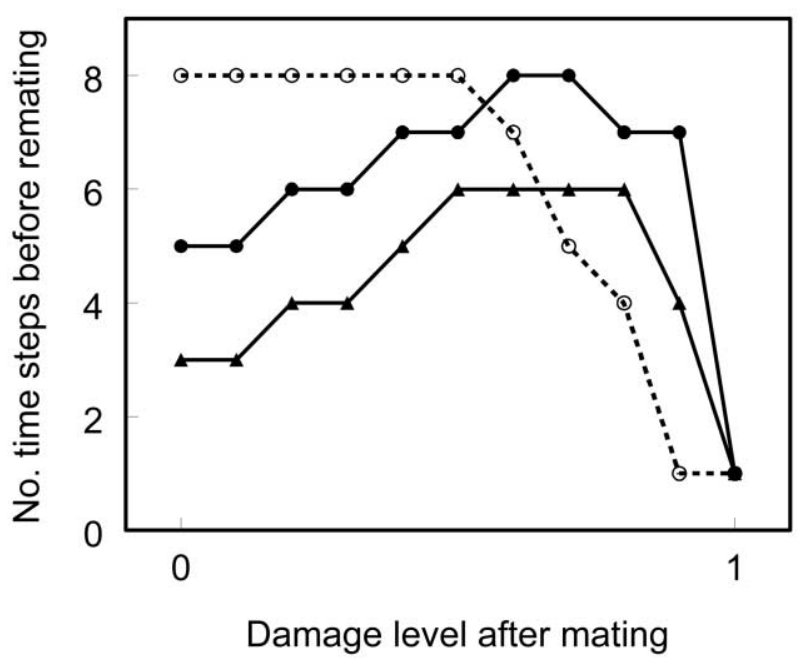

Figure 10: Remating interval at the evolutionarily stable strategy in relation to the damage level after mating. Results are from models in which both mating and oviposition rate are decided by the females (open circles, broken line), or females decide only whether to mate (filled symbols, solid lines). Parameter values: for all lines, $i_{\max }=8, a=5$, and $b=2$; for circles, $c=5$; for triangles, $c=3$; and for solid lines and filled circles, $E=0.5$.

of mating and oviposition, predicts an ESS with an intermediate amount of damage (fig. 11), although numerically the amount of damage tends to be less than in the first model with the same parameter values (see table 1). The major difference from the first model is that terminal damage leading to a semelparous female life history is never an ESS. This is understandable, because in the first model, females lay eggs (and die as a result of the terminal investment) before the deleterious effects of a male's damage on his own reproductive success can be felt. In contrast, when oviposition precedes mating within a time step, mated females must survive to the next time step before making any potentially terminal investment. In this case, a high level of damage by a male will impinge on the female's survival to oviposit and hence on his own reproductive success. It should be noted that, as before, females make a terminal investment in the last step of their life histories (except when the male population is nondamaging). This must be distinguished from terminal damage by males, which leads to semelparity. As in the first model, a nondamaging population of males can always be invaded by a damaging mutant. Because females remate at every time step in a nondamaging population and because optimal oviposition rates always increase with increasing levels of damage, in the first model it is inevitable that damaging mutants have higher fitness (see above). However, if females first have to survive to the next time step, the reduced survival of a female mated by a damaging male might potentially outweigh the benefits that the male enjoys through increased oviposition rate. With the fitness functions used in the models presented here, this seems not to be the case, although it is not clear whether that would be true for all fitness functions with the same general shape. As in the first model, the optimal female strategy implies that oviposition rates increase but that remating intervals decrease, with increasing levels of damage: so as before, male damage evolves because of its effect on oviposition rate and despite its effect on remating interval.

\section{Discussion}

The most important result of the modeling presented here is that, when females adaptively modify their mating and oviposition behavior in relation to male damage, some level of male damage (greater than 0) is always an ESS. Nondamaging behavior is not an ESS because damaging mutants always invade a nondamaging population. The model, however, does not work quite as suggested in the introduction: at the ESS, females do indeed increase their oviposition rate with increasing levels of accumulated damage after mating, but the remating interval actually decreases rather than increases as suggested by the verbal model. In other words, when females decide both oviposition rate and whether to remate, male mating damage evolves because of its effect on oviposition rate and despite its effect on remating interval (as in the simplified example in fig. 1D).

Male mating damage is also an ESS in models in which females decide only oviposition rate or whether to mate. The result for a model in which females decide only oviposition rate are broadly similar to those of the full model (except, of course, that remating interval does not vary with damage). If females decide only whether to remate, damage is also an ESS but is selectively neutral in a nondamaging population. However, as soon as the population level of damage drifts above 0 , the population would converge on the damaging ESS. In this model, remating interval increases and then decreases with a female's level of accumulated damage after mating. Johnstone and Keller (2000) also consider the case in which females decide only whether to remate. The results reported here differ from their conclusions in that here a damaging ESS does not require an accelerating cost of damage (at least in terms of survival between time steps) and zero male damage is never an equilibrium with convergence stability. Explanations for the lack of convergence stability for zero damage are given in "Results" above.

In models in which mating occurs before oviposition within a time step, males benefit from increased damage in terms of increased oviposition rate for one time step 
(A)

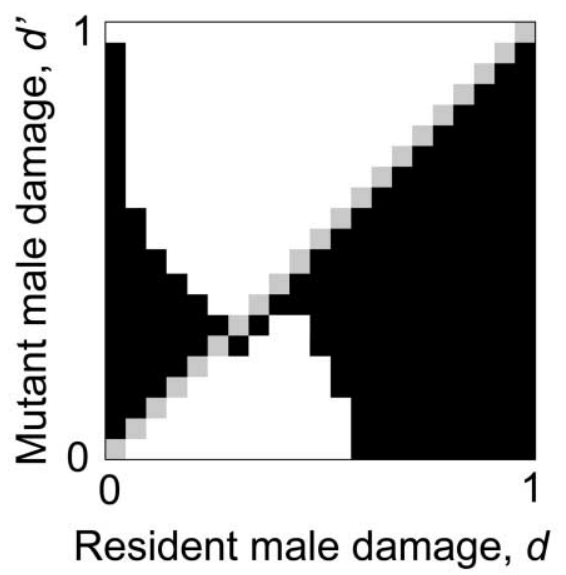

(B)

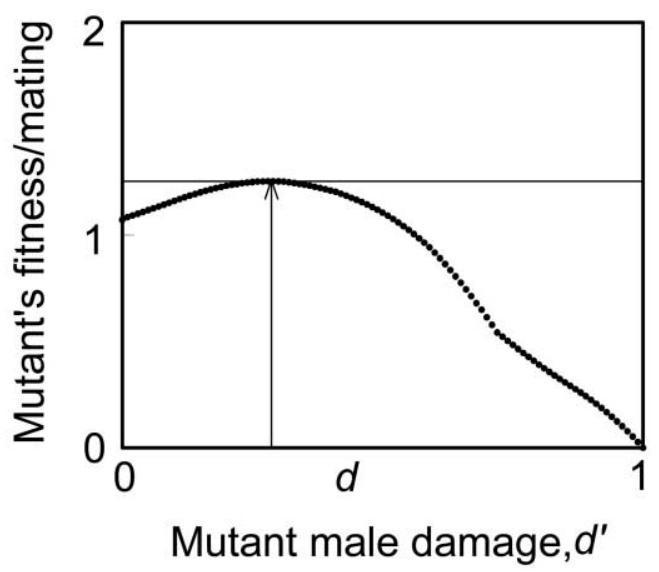

Figure 11: Evolutionarily stable strategy male damage in a model in which both mating and oviposition rate are female decisions, but in which mating takes place after, not before, oviposition in each time step. Parameter values are $i_{\max }=9$ (and fertility, $Y$, calculated from eq. [20]), $a=$ $5, b=2$, and $c=5$.

before the male's damage rebounds on his own fitness by reducing the female's survival. They thus maximize the chance that mating damage will be evolutionarily stable. In models in which mating occurs after oviposition within a time step, the deleterious effects of damage on the female's survival are not delayed in this way. Unsurprisingly, comparisons of models with each of the two sequences show that terminal damage is only an ESS when the costs of damage are delayed. However, the predictions of the models are otherwise remarkably similar and show that in general the evolutionary stability of intermediate levels of damage does not depend on such a delay. Moreover, the effect of shortening the time step in either type of model is likely to produce predictions that are intermediate between those for the two sequences of behavior because mating and oviposition can be thought of as occurring more and more simultaneously. This argues that the evolutionary stability of male mating damage is not simply a consequence of the discrete time steps in the models presented here but would also be an outcome of a model with continuous time.

One feature of the models in which females decide oviposition rate (or whether they also decide whether to mate) is that, for certain parameter values, terminal damage that provokes females into terminal reproductive investment and hence generates a semelparous life history is an ESS. In the absence of male mating damage, these female optimal life histories would be iteroparous. Such species with terminal damage by males should have iteroparous males and semelparous females. While examples of such a life history do not immediately spring to mind, the existence of an ESS with terminal damage does emphasize that sexual conflict can potentially have profound qualitative effects on the evolutionarily stable life history.

The models make a number of simplifying assumptions for the sake of tractability. For example, they assume that survival or fertility drops to zero as oviposition rate, damage, or time since mating increase. The fact that survival drops to zero with increasing oviposition rate means that it is possible for females to make terminal reproductive investment. Without this assumption, terminal damage cannot be an ESS. On the other hand, the assumption that survival in relation to damage and fertility drops to zero probably does not greatly affect the outcome because females make terminal reproductive investment before this becomes inevitable because survival has dropped to zero, and they often remate before this becomes inevitable because of zero fertility. The models also assume that there is complete last male precedence, and as shown by Johnstone and Keller (2000), this increases the evolutionarily stable level of male mating damage. However, under any kind of sperm precedence except complete first male precedence (both biologically implausible and removing the sexual conflict that lies behind male mating damage), male paternity drops when the female remates. There is still, therefore, selection for increasing oviposition rate in the time steps immediately following mating and delaying remating, although not as strong as with complete last male sperm precedence. Finally, the model assumes that inflicting damage has no direct cost to the male. Such costs will make zero damage more likely either as an alternative ESS to some nonzero level of damage or as the sole ESS. 
The models suggest that there is no impediment to the initial invasion of damaging mutants into a nondamaging population, but it is reasonable to ask whether that is really so: the females' response to damage that increases male fitness depends on the females having a strategy matrix already "built in" by selection that tells them how to modify their life history in relation to damage. But in a nondamaging population, females would never have experienced the damage that would have been built in the matrix and so are not expected to show phenotypic plasticity in response to it. Thus, damaging behavior might not invade initially so easily if it were dependent on the response to the specific kind of damage caused by males. If, however, females had some general way of monitoring their residual reproductive value, they might well respond to damage by modifying their life histories, and male mating damage could invade a nondamaging population. Such a system of monitoring residual reproductive value would presumably involve hormonal signaling. If females do have such a signaling system, males would be selected to manipulate female life histories by manipulating the hormonal signal rather than by damaging the female. That way they would gain from the female life-history modifications but not suffer from the actual decrease in female fitness caused by the damage.

Finally, the models restrict the ways in which each sex can respond evolutionarily: males can only manipulate females by inflicting damage on them, and females can only limit the consequences of damage by modifying their subsequent life history. Clearly, however, we would expect selection for females to limit the extent of damage (Rice 1996). This in turn would lead males to inflict damage in ways that are difficult to avoid. Drosophila males, for example, transfer seminal fluid before transferring sperm when mating (Harshman and Prout 1994). Females cannot therefore limit any damage by limiting the transfer of seminal fluid. This observation, however, is also consistent with the pleiotropy hypothesis, with seminal proteins manipulating oviposition rate and remating directly. If males could manipulate oviposition rate or remating directly, what would be the ESS, assuming that none of the manipulations has any direct cost to the male? If males can manipulate remating interval, then their best strategy is to prevent the female from ever remating (even after a time when her fertility using his sperm has dropped to zero). In this situation, sexual conflict over the female life history disappears, and the life history that maximizes the female's fitness also maximizes the male's fitness. It is then unnecessary for the male to manipulate oviposition rate directly, and any damage that he inflicts could only reduce his own fitness. The rarity of such life histories suggests that it is difficult for males to prevent females from ever remating. If males can manipulate oviposition rate (but not remating interval), the evolutionary outcome is not so straightforward. In particular, unlike the reverse scenario, sexual conflict is not abolished by a male imposing his own optimal oviposition rate. Further modeling would then be needed to investigate whether some level of male damage could then be evolutionarily stable because of its indirect effect on remating interval.

Distinguishing experimentally between alternative models for the evolution of male mating damage is a challenge. In the fly Sepsis cynipsea, in which mechanical damage is inflicted on females during copulation, females that have mated more previously are more willing to remate but have unchanged fecundity (Hosken et al. 2003). The increase in the female propensity to remate is consistent with the evolutionary response predicted in the models presented here, but the damaging males appear to lose rather than gain fitness, suggesting that male damage is a deleterious pleiotropic effect (Hosken et al. 2003). Females of three other insect species that were experimentally damaged after copulation had unchanged remating probabilities and unchanged or reduced fecundity relative to undamaged controls (Morrow et al. 2003). In other cases, interpretation will be more difficult. For example, seminal proteins that increase oviposition rate might directly manipulate the female's oviposition control mechanism, with a reduction in survival as a deleterious side effect, or they might constitute toxins that, by reducing survival, provoke the female into increasing her oviposition rate. In either case, what we might expect to see now is the seminal protein triggering some kind of signaling system that leads to an increase in oviposition rate. Nevertheless, the models presented here clearly show that male mating damage, whose only direct effect is to decrease female survival, can potentially evolve because it stimulates the female to modify her life history in a way that also increases male fitness. Testing whether they actually do so is the next step in the puzzle.

\section{Acknowledgments}

I wish to thank W. Mooij for help getting started with programming in $\mathrm{C}++$; J. Koene and M. Visser for helpful comments on an earlier draft; L. Kvarnemo, T. Tregenza, and $\mathrm{N}$. Wedell for organizing the symposium on sexual conflict and life histories at Tovertorp; and D. Hosken and R. Snook for organizing the symposium on sexual conflict at Leeds and the publication of this symposium issue of the American Naturalist.

\section{Literature Cited}

Chapman, T., L. F. Liddle, J. M. Kalb, M. F. Wolfner, and L. Partridge. 1995. Cost of mating in Drosophila melanogaster females is mediated by male accessory gland products. Nature 373:241-244. 
Chapman, T., D. M. Neubaum, M. F. Wolfner, and L. Partridge. 2000. The role of male accessory gland protein Acp36DE in sperm competition in Drosophila melanogaster. Proceedings of the Royal Society of London B 267:1097-1105.

Chapman, T., G. Arnqvist, J. Bangham, and L. Rowe. 2003. Sexual conflict. Trends in Ecology \& Evolution 18:41-47.

Charnov, E. L., and J. R. Krebs. 1974. On clutch size and fitness. Ibis 116:217-219.

Chen, P. S., E. Stumm-Zollinger, T. Aigaki, J. Balmer, M. Bienz, and P. Böhlen. 1988. A male accessory gland peptide that regulates reproductive behavior in female D. melanogaster. Cell 54:291-298.

Constantz, G. D. 1984. Sperm competition in poeciliid fishes. Pages 465-485 in R. L. Smith, ed. Sperm competition and the evolution of animal mating systems. Academic Press, London.

Crudgington, H. S., and M. T. Siva-Jothy. 2000. Genital damage, kicking and early death. Nature 407:855-856.

Gems, D., and D. L. Riddle. 1996. Longevity in Caenorhabditis elegans reduced by mating but not gamete production. Nature 379:723725.

Geritz, S. A. H., É. Kisdi, G. Meszéna, and J. A. J. Metz. 1998. Evolutionarily singular strategies and the adaptive growth and branching of the evolutionary tree. Evolutionary Ecology 12:3557.

Harshman, L. G., and T. Prout. 1994. Sperm displacement without sperm transfer in Drosophila melanogaster. Evolution 48:758-766.

Hosken, D. J., O. Y. Martin, J. Born, and F. Huber. 2003. Sexual conflict in Sepsis cynipsea: female reluctance, fertility and mate choice. Journal of Evolutionary Biology 16:485-490.

Johnstone, R. A., and L. Keller. 2000. How males can gain by harming their mates: sexual conflict, seminal toxins, and the cost of mating. American Naturalist 156:368-377.
Lessells, C. M. 1999. Sexual conflict in animals. Pages 75-99 in L. Keller, ed. Levels of selection in evolution. Princeton University Press, Princeton, NJ.

Mangel, M., and C. W. Clark. 1988. Dynamic modeling in behavioral ecology. Princeton University Press, Princeton, NJ.

Michiels, N. K. 1998. Mating conflicts and sperm competition in simultaneous hermaphrodites. Pages 219-254 in T. R. Birkhead and A. P. Møller, eds. Sperm competition and sexual selection. Academic Press, London.

Morrow, E. H., G. Arnqvist, and S. Pitnick. 2003. Adaptation versus pleiotropy: why do males harm their mates? Behavioral Ecology 6:802-806.

Parker, G. A. 1979. Sexual selection and sexual conflict. Pages $123-$ 166 in M. S. Blum and N. A. Blum, eds. Sexual selection and reproductive competition in insects. Academic Press, New York.

Price, C. S. C., K. A. Dyer, and J. A. Coyne. 1999. Sperm competition between Drosophila males involves both displacement and incapacitation. Nature 400:449-452.

Rice, W. R. 1996. Sexually antagonistic male adaptation triggered by experimental arrest of female evolution. Nature 381:232-234.

2000. Dangerous liaisons. Proceedings of the National Academy of Sciences of the USA 97:12953-12955.

Snook, R. R., and D. J. Hosken. 2004. Sperm death and dumping in Drosophila. Nature 428:939-941.

Trivers, R. L. 1972. Parental investment and sexual selection. Pages 136-179 in B. Campbell, ed. Sexual selection and the descent of man. Aldine, Chicago.

Williams, G. C. 1966. Natural selection, the costs of reproduction and a refinement of Lack's principle. American Naturalist 100: $687-690$. 